\title{
Magnetic Resonance Imaging-Guided versus Surrogate-Based Motion Tracking in Liver Radiation Therapy: A Prospective Comparative Study
}

\author{
Chiara Paganelli, MSc, ${ }^{*}$ Matteo Seregni, PhD, ${ }^{*}$ Giovanni Fattori, PhD, * Paul \\ Summers, PhD, ${ }^{\dagger}$ Massimo Bellomi, MD, ${ }^{\dagger, \ddagger}$ Guido Baroni, PhD, ${ }^{*, \delta}$ and Marco \\ Riboldi, PhD*,§
}

\begin{abstract}
*Dipartimento di Elettronica, Informazione e Bioingegneria, Politecnico di Milano, Milano, Italy; ${ }^{\dagger}$ Division of Radiology, Istituto Europeo di Oncologia, Milano, Italy; ${ }^{\ddagger}$ Department of Health Sciences, Università degli Studi di Milano, Milano, Italy; and ${ }^{\S}$ Bioengineering Unit, CNAO Foundation, Pavia, Italy
\end{abstract}

\author{
Summary \\ We compared magnetic \\ resonance imaging (MRI)- \\ guided tracking to surrogate- \\ based tracking in 30 subjects, \\ relying on MRI cine imaging \\ and automatic feature \\ extraction. Results show that \\ the gain achieved with MRI \\ guidance compared to that of \\ surrogate-based tracking is \\ dependent on the tracking \\ error tolerance and intrinsic \\ motion features. The pro- \\ posed method can provide \\ spatially distributed and \\ clinically valuable motion \\ information which could \\ support identification of \\ patient-specific optimal \\ tracking strategies.
}

\begin{abstract}
Purpose: This study applied automatic feature detection on cine-magnetic resonance imaging (MRI) liver images in order to provide a prospective comparison between MRI-guided and surrogate-based tracking methods for motion-compensated liver radiation therapy.

Methods and Materials: In a population of 30 subjects ( 5 volunteers plus 25 patients), 2 oblique sagittal slices were acquired across the liver at high temporal resolution. An algorithm based on scale invariant feature transform (SIFT) was used to extract and track multiple features throughout the image sequence. The position of abdominal markers was also measured directly from the image series, and the internal motion of each feature was quantified through multiparametric analysis. Surrogate-based tumor tracking with a state-of-the-art external/internal correlation model was simulated. The geometrical tracking error was measured, and its correlation with external motion parameters was also investigated. Finally, the potential gain in tracking accuracy relying on MRI guidance was quantified as a function of the maximum allowed tracking error.

Results: An average of 45 features was extracted for each subject across the whole liver. The multi-parametric motion analysis reported relevant inter- and intrasubject variability, highlighting the value of patient-specific and spatially-distributed measurements. Surrogate-based tracking errors (relative to the motion amplitude) were were in the range $7 \%$ to $23 \%(1.02-3.57 \mathrm{~mm})$ and were significantly influenced by external motion
\end{abstract}

Reprint requests to: Chiara Paganelli, MSc, Dipartimento di Elettronica, Informazione e Bioingegneria, Politecnico di Milano, 20133 Milano, Italy.; E-mail: chiara.paganelli@polimi.it
The work was supported by Italian Association for Cancer Research (AIRC), study n. R603-IEO S648/511.

Conflict of interest: none. 
parameters. The gain of MRI guidance compared to surrogate-based motion tracking was larger than $30 \%$ in $50 \%$ of the subjects when considering a $1.5-\mathrm{mm}$ tracking error tolerance.

Conclusions: Automatic feature detection applied to cine-MRI allows detailed liver motion description to be obtained. Such information was used to quantify the performance of surrogate-based tracking methods and to provide a prospective comparison with respect to MRI-guided radiation therapy, which could support the definition of patientspecific optimal treatment strategies.

\section{Introduction}

During the last decades, several developments in image guided radiation therapy were motivated by the need to improve treatment conformity for tumors affected by respiration-induced anatomical changes, which suffer from poor targeting accuracy $(1,2)$. For this purpose, different strategies for motion mitigation were investigated and implemented on modern treatment units, relying on limiting (3), monitoring (4), or tracking target motion (5). For all of these approaches, accurate quantification of res-piratory motion is important for the overall treatment effectiveness (6), especially when motion mitigation relies on external surrogate-driven motion models (7), as imple-mented in several commercial systems $(8,9)$. Such a requirement applies to both the planning and treatment delivery stages, as the effects of motion need to be accurately simulated and consistently verified throughout treatment. Specifically in radiation therapy applications $(10,11)$, the current clinical practice considers 4-dimensional computed tomography (4DCT) (12) to be the standard imaging modality for timeresolved acquisition. However, the single average breathing cycle provided by $4 \mathrm{DCT}$ does not properly capture intercycle variability and respiratory motion irregularities that occur on a daily basis (7). Fast dynamic magnetic resonance imaging (MRI) can overcome such limitations, allowing acquisition of a significant amount of breathing cycles at high temporal resolution and better soft tissue contrast $(2,13)$. These unique features also motivated recent technological de-velopments toward integration of MRI with radiation therapy treatment units, aiming at the implementation of fully MRI-guided treatments (14-17). In terms of guidance during treatment to account for breathing motion, the use of low latency cineMRI images has been proposed (18-20).

Specifically, the use of 2D cine-MRI (21) for organ motion quantification has been widely reported in published reports. Different studies $(2,21,22)$ verified the capability of cine-MRI to assess tumor mobility and local motion. In the treatment of liver lesions, a target displacement in the range of 12 to $26 \mathrm{~mm}$ in the superior-inferior (SI) anatomical axis has been reported (23), relying on image registration of cine-MRI images. Alternatively, manual anatomical landmarks (ie features) have been considered for motion quantification (24-27), with craniocaudal liver displacement measured in the ranges of 6.9 to
$35.4 \mathrm{~mm}$ (26) and 7.8 to $22.8 \mathrm{~mm}$ (27). Recently, Dowling et al (28) proposed a method to track manual points through multiple cine-MRI slices. Similarly, a single internal structure (ie vessel) was tracked on cine MRI images by template matching from a manually selected region of interest in $(18,29)$. Other studies in lung proposed analogous solutions for motion tracking of anatomical structures (30, $31)$. However all these approaches are time consuming and suffer from interoperator variability. As an alternative, several algorithms for automatic feature extraction have been developed, such as the scale invariant feature transform (SIFT). Such a method is widely reported in the literature for image registration purposes $(32,33)$ and, as mainly required in radiation therapy applications, for organ motion quantification $(34,35)$.

In this work, we proposed a prospective comparative study where cine-MRI images were analyzed to quantify the potential benefit of real-time MRI guidance during treatment. Multiple spatially distributed features were extracted and tracked in cine-MRI image series from 30 subjects by means of a SIFT-based method, providing accurate motion quantification as a function of breathingrelated variables. Finally, we compared MRI-guided liver motion tracking with a state-of-the-art tracking approach based on external surrogates, aiming at patient stratification based on the expected accuracy improvement that could be obtained by applying MRI guidance.

\section{Methods and Materials}

\section{Dataset}

An MRI protocol was developed to simultaneously capture liver motion and movement of external fiducial markers (ie cod liver oil capsules, $13 \mathrm{~mm}$ long $\times 4 \mathrm{~mm}$ radius). Subjects were placed in the scanner in the supine position and external markers were placed on the thoracoabdominal surface in a $3 \times 3$ (or $3 \times 4)$ configuration.

We acquired $2 \mathrm{D}$ cine-MRI images in 30 subjects (5 volunteers and 25 patients) at the European Istitute of Oncology (Milano, Italy) with a 1.5-T scanner (Magnetom Avanto; Siemens Medical System). All subjects participated by signing informed consent forms (study no. R603-IEO S648/ 511). A balanced steady-state free precession sequence (TrueFISP on the Siemens platform) was used during free breathing to acquire 2 interleaved oblique sagittal slices of 
the liver in 240 frames (ie 120 frames/slice during a period of 74.4 seconds). We selected and oriented the 2 slices by acquiring 2 marker columns, respectively, and including liver tumor (when present), as shown in Figure 1A. MRI sequence parameters were optimized to ensure satisfactory time sampling for the respiratory cycle description and adequate spatial resolution for the proper identification of internal structures, as follows: repetition time/echo time: 308.59 msec/1.22 msec; flip angle: $65^{\circ}$; bandwidth: $977 \mathrm{~Hz} /$ pixel; scan matrix: $256 \times 256$ pixels, $1.28-\times 1.28-\mathrm{mm}$ spacing, $10-$ $\mathrm{mm}$ slice thickness; acquisition time: $310 \mathrm{msec} / \mathrm{slice}$.

\section{Marker localization}

The external marker localization was based on the Hough transform for circular shapes (36). We identified the marker centers, as follows: manual selection of a region of interest (ROI) around each visible marker in the first frame for each slice; and application of the Hough transform in the same ROI of all frames with the marker radius set in the range 2.56 to $5.12 \mathrm{~mm}$ (to consider the variability of the capsule section in the oblique acquisition modality) (Fig. 1B).

We tested the accuracy of the method by using static uniformity and linearity phantoms (spatial resolution of $0.72 \times 0.72 \times 4 \mathrm{~mm})(37)$, with known distances between markers $(60 \mathrm{~mm})$.

\section{Internal feature extraction}

A SIFT-based method (35) was used to extract and match internal features from the image frames, as proposed previously (34). Specifically, for each slice, we applied SIFT to image frame pairs by keeping the first frame as reference. Final trajectories resulted from those features actually tracked on all frames and reporting less than 20 misses, which were replaced by cubic interpolation. Furthermore, trajectories with a motion range less than twice the pixel size were rejected, whereas outliers in the final trajectories due to wrong feature matching were excluded and replaced through interpolation. Outliers were identified as data points with a distance from the trajectory centroid greater than a threshold, defined as

$$
\text { threshold }=d_{75^{\text {th }}}+1.5 \times d_{75^{\text {th }}-25^{\text {th }}}
$$

where $d_{75^{\text {th }}}$ is the 75 th percentile of the points-to-centroid distance distribution $d$, whereas $d_{75^{\text {th }}-25^{\text {th }}}$ is the IQR.

In order to describe the spatial distribution of detected features, distances between each trajectory centroid and their overall center of mass were measured. For each subject, the resulting distance distribution was quantified with median value, interquartile range (IQR), and 5th and 95th percentiles.

\section{Motion quantification}

In order to handle interleaved acquisition of MRI slices, internal and external motion trajectories were resampled at $3.33 \mathrm{~Hz}$ (ie information from the 2 slices was temporally aligned). Trajectories belonging to different features were analyzed by considering the following parameters: main motion direction with respect to the SI axis; average peakto-peak amplitude; average respiratory period; and phase shift with respect to the external abdominal motion.

Distributions of these parameters, computed individually for each of the detected liver features, were grouped in subject-specific measurements, where the central tendency was expressed in terms of median value (subject-specific average), and the dispersion was expressed as IQR and 95th percentile (38).

\section{Surrogate-based internal motion tracking}

A state-of-the-art motion mitigation strategy based on surrogate-driven tumor tracking was considered (39). Such an approach relies on a state-augmented quadratic model to relate the internal target motion (ie liver features) with the external surrogates (abdominal markers):

$$
I(s)=a s^{2}+b s+c+d s^{2}+e \dot{s}
$$

where $s$ is the external surrogate and $I(s)$ is the estimated target position. The model parameters $[a, b, c, d, e]$ were optimized through a least-squares algorithm applied to a training dataset
A

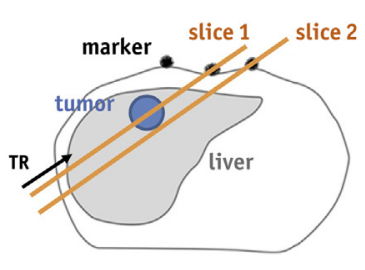

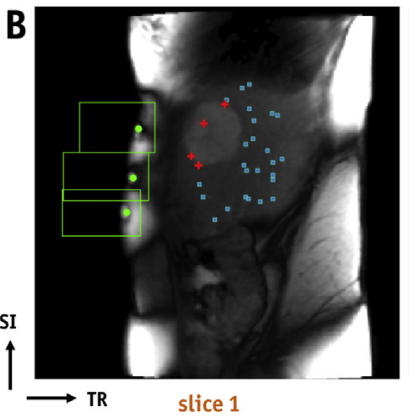

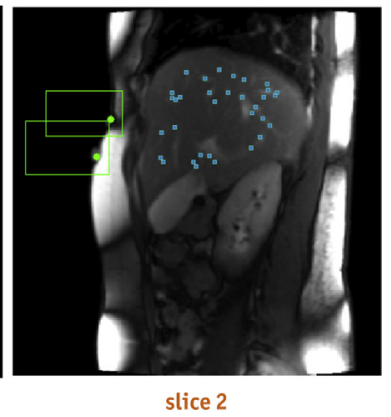

Fig. 1. MRI protocol and feature identification. (a) Slice selection and orientation according to markers and tumor position. (b) External marker localization (green circles with the respective squared ROI) and internal feature detection (light blue squares for healthy liver and red crosses for tumor). MRI = magnetic resonance imaging; ROI $=$ region of interest; SI = superior-inferior; $\mathrm{TR}=$ transversal. 
consisting of the first 65 samples (20 seconds) of the cine-MRI signal. The remaining samples (approximately 50 seconds) were used to measure the tracking accuracy.

The feature tracking error $\left(T E^{f}\right)$ was measured as the Euclidean distance between the estimated and corresponding actual motion samples in the testing dataset. Subject-specific results were obtained by computing root mean square $\left(T E_{r m s}^{s}\right)$, median value $\left(T E_{\text {med }}^{s}\right)$, IQR $\left(T E_{i q r}^{s}\right)$, and 95th percentile $\left(T E_{95}^{s}\right)$ of the $T E^{f}$ distributions. Finally, tracking error distribution was also expressed as a percentage of each feature 2D range of motion: their RMS is referred to as $T E_{\%}^{s}$.

\section{Surrogate-based versus MRI-guided tracking}

The subject-specific gain in accuracy when using MRI guidance, with respect to external and internal correlation models, was quantified through the following index:

$$
M R I_{\text {gain }}\left(T E_{t h}\right)=100 \frac{N\left(T E>T E_{t h}\right)}{N_{\text {tot }}}[\%]
$$

where $N\left(T E>T E_{t h}\right)$ is the number of samples whose tracking error is larger than a given threshold $\left(T E_{t h}[\mathrm{~mm}]\right)$ and $N_{t o t}$ is the total number of samples. For each subject, $M R I_{\text {gain }}$ was computed for $T E_{t h}$ values in the range $[0,0.1, \ldots, 5] \mathrm{mm}$. Specifically, $M R I_{\text {gain }}=0 \%$ means that the required tracking accuracy $\left(T E_{t h}\right)$ can be fully achieved by using surrogate-based tracking, without any improvement provided by MRI guidance. Conversely, the higher the $M R I_{\text {gain }}$ value the more relevant the increase is in the tracking accuracy that can be expected if direct target localization through MRI guidance is applied instead of surrogate-based tracking.

\section{Surrogate-based tracking performance}

To further investigate the performance of the surrogatebased tracking method, the correlation between the percentage tracking errors $\left(T E_{\%}^{s}\right)$ and the parameters related to external motion, which are the absolute phase shift $\left(|\Delta \varphi|_{\text {med }}^{s}\right)$, the number of external markers used as surrogates $(\mathrm{m})$, and their average correlation with the internal feature motion $(\rho)$, was quantified through the Pearson correlation coefficient $\left(R_{|\Delta \varphi|}, R_{m}\right.$, and $R_{\rho}$, respectively), considering $p-$ value $\leq 0.01$ for statistical significance.

\section{Results}

\section{Features and markers localization}

The use of SIFT in a CT image of a phantom (Rando, Phantom Laboratories, Salem, NY) has already been reported (33), with an error within the voxel dimension $(1.87 \times 1.87 \times 3 \mathrm{~mm})$. The number of features localized in each subject was between 5 and 99 . On average, the distance from their center of mass was $25.4 \pm 34.4 \mathrm{~mm}$ (median $\pm \mathrm{IQR}$ ). The marker localization error, measured on the uniformity and linearity phantom, was $0.46 \pm 0.72 \mathrm{~mm}$ (mean $\pm \mathrm{SD}$ ), ie smaller than the phantom resolution.

\section{Motion quantification}

The main motion direction (Fig. 2 A) was oriented $-17^{\circ}$ with respect to the SI anatomical axis (ie mainly cranial with a small transversal component). Intrasubject variability, expressed as IQR, was reported from $2.19^{\circ}$ up to $18.80^{\circ}$. Phase shifts (Fig. 2 B) with respect to the external abdominal motion showed a relevant intersubject variability $\left(-80.77^{\circ},+19.96^{\circ}\right)$, and in 4 patients (Fig. 2, p12, p13, p17, and p20), high intrasubject variations were also reported (IQR up to $67.40^{\circ}$ ).

Median values of peak-to-peak motion amplitude measured between $3.10 \mathrm{~mm}$ and $24.02 \mathrm{~mm}$, with an intrasubject variability ranging from $0.48 \mathrm{~mm}$ to $4.15 \mathrm{~mm}$ (Fig. 2 C). Median motion periods (Fig. 2 D) ranged from 2.61 to 7.48 seconds, with very limited intrasubject variability $(<0.83$ second $)$.

\section{Surrogate-based versus MRI-guided tracking}

Figure 3 shows the gain of MRI guidance versus external/ in-ternal correlation for the 30 subjects, evaluated for different error thresholds in the range of 0 to $5 \mathrm{~mm}$. Relevant inter-subject variability can be observed. For instance, if the error threshold (ie maximum allowed error) is set at $1.5 \mathrm{~mm}, M R I_{\text {gain }}$ ranges between $11.78 \%$ (Fig. 2, p9) and 68.13\%(Fig. 2, p21; median $=29.08 \%$; $\mathrm{IQR}=17.59 \%)$. If the required accuracy is $2 \mathrm{~mm}, M R I_{\text {gain }}$ is larger than $50 \%$ in only 2 subjects. However, if better accuracy is required (ie $1 \mathrm{~mm}$ ), 17 subjects exhibit a gain due to MRI guidance $>50 \%$.

\section{Surrogate-based tracking performance}

Figure 4 shows the tracking errors and their correlations with external motion parameters. TE showed both interfeature and intersubject variability. Interfeature variability can be observed in the $T E_{i q r}^{s}$ values, which were measured from $0.68 \mathrm{~mm}$ up to $2.96 \mathrm{~mm}$.

As an example, Figure 5 shows the $T E_{r m s}^{f}$ map (Fig. 4, p14) and motion traces corresponding to the features with the highest and lowest errors. The time-resolved TE map is available in Figure E1 (supplementary material can be found at www.redjournal.org).

The $T E_{r m s}^{s}$ was found between 1.02 and $3.57 \mathrm{~mm}$ (subject population average $=1.76 \mathrm{~mm}$ ); $T E_{\text {med }}^{s}$ was lower than the image spatial resolution in 23 out of 30 subjects. The intersubject variabjlity can be observed in the percentage tracking errors $T E^{s}$, which ranged between approximately $7 \%$ and $23 \%$ (population average $=13 \%$ ) of the $2 \mathrm{D}$ motion amplitude (Fig. 4A). 

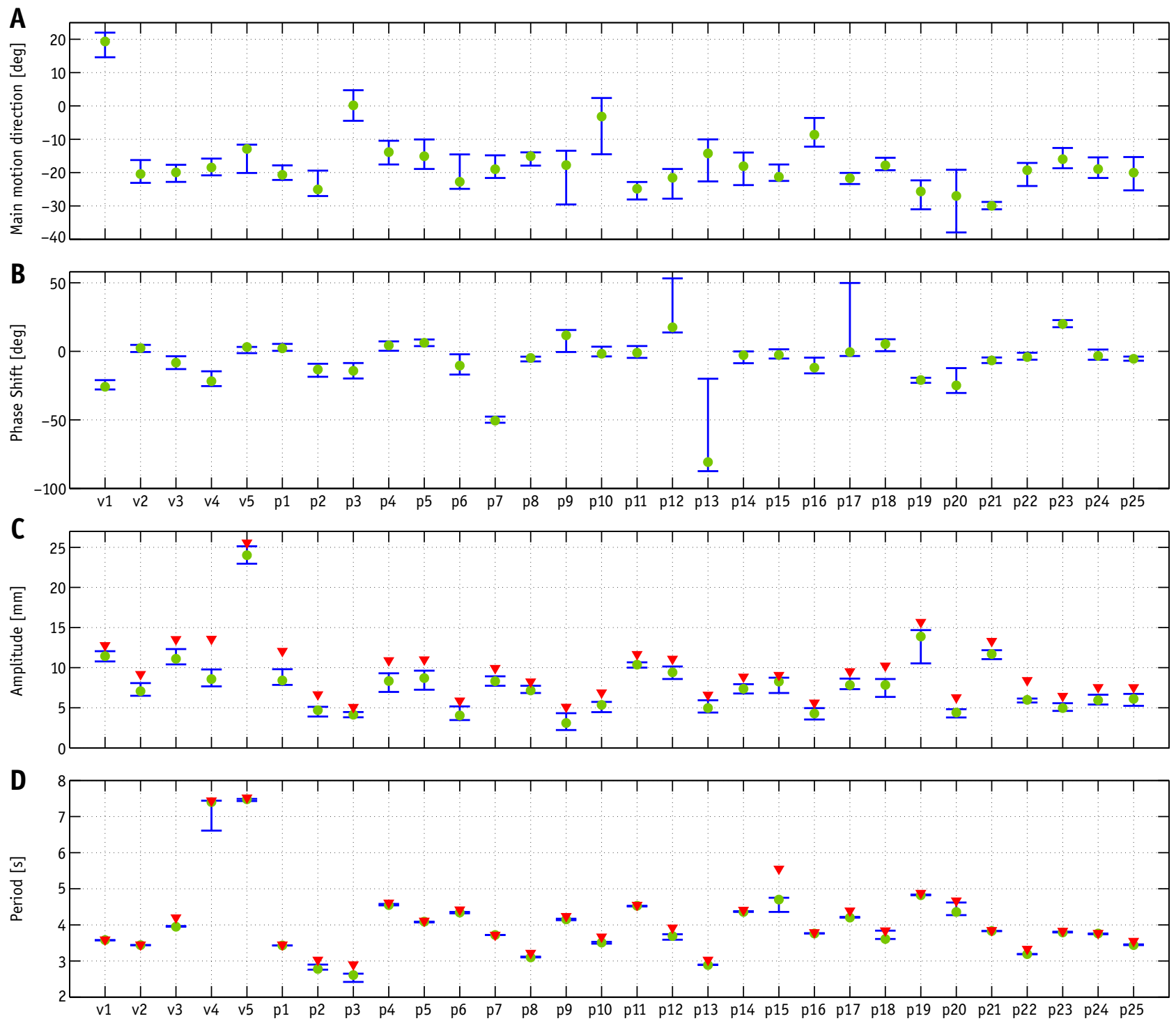

Fig. 2. Motion quantification parameters expressed as medians (green circles), IQRs (blue bars), and 95th percentiles (red squares for motion amplitude and period only). (A) Main motion direction. (B) Phase-shift; (C) motion amplitude; and (D) period. $\mathrm{IQR}=$ interquartile range.

The number of external markers used as surrogates $(\mathrm{m})$ was between 1 and 7 , with an average internal-external correlation $(\rho)$ between 0.40 and 0.90 (Fig. 4B). As expected, significant (negative) correlations between these parameters and the percentage tracking error were measured: $\left(R_{m}=-0.47, p<10^{-3}, R_{\rho}=-0.62, p<10^{-2}\right)$.

Finally, phase shifts significantly degraded the tracking accuracy: $R_{|\Delta \varphi|}=0.52, p<10^{-2}$, as seen in Figure 4B.

\section{Discussion}

In this work, an automatic feature extraction method was applied to cine-MRI images of the liver, first, to study the performance of a surrogate-based tracking method, and second, to prospectively investigate the improvement in accuracy that could be obtained by applying MRI guidance for motion tracking.

\section{Feature extraction}

A SIFT-based method (35) was proposed to extract and track features in liver cine-MRI series. The large number of features (45 on average) and spatial distribution provided detailed liver motion information within the selected slices. Although the interleaved acquisition of two 1-cm-thick oblique slices provided only 2D motion information, such imaging modalities were selected to combine adequate inplane resolution $(1.28 \mathrm{~mm})$ with a slice acquisition frequency $(1 / 620 \mathrm{msec})$ able to sample respiratory acts as short as 1.3 seconds. Future developments concerning 3D 


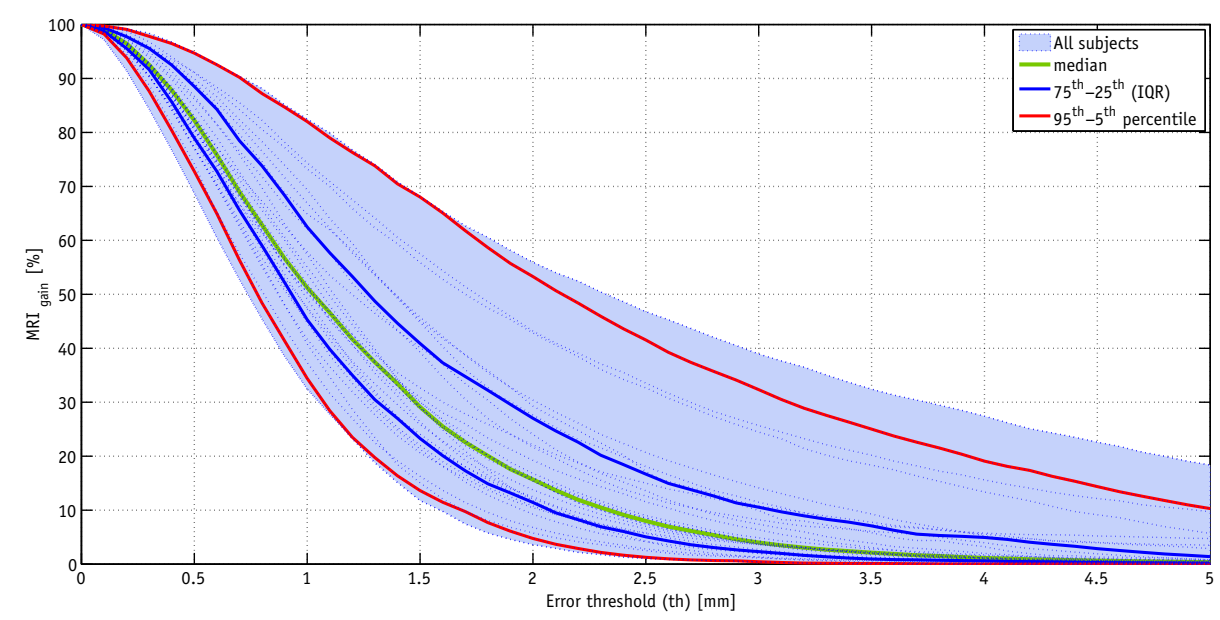

Fig. 3. Gain of MRI guidance for the 30 subjects (dotted curves). Median, 75th to 25th, and 95th to 5th percentile curves are shown in green, blue, and red respectively. MRI = magnetic resonance imaging.

point reconstruction from 2D MRI images should be considered, as proposed previously $(18,19,29)$. However, unlike landmark tracking methods based on template matching $(18,29)$, SIFT is invariant to rigid and nonrigid transforms (33), thus ensuring robust feature tracking throughout the cine-MRI sequence even in the presence of deformations and/or rotations. Moreover, the proposed approach requires minimal user interaction and limited imaging and processing time (less than 30 minutes overall, 14 to 20 minutes for feature extraction and 5 to 7 minutes to reconstruct trajectories), both compatible with the clinical routine. Finally, differently from a previous study (18), the external surrogate signal was derived directly from the image series, not requiring any external monitoring device and providing technical and procedural simplifications to the clinical workflow.

\section{Motion quantification}

Liver motion parameters were measured in a large subject population, relying on multiple features, thus obtaining inter- and intrapatient variability information. In-plane motion direction was mainly craniocaudal $\left(+20^{\circ}<\alpha<\right.$ $-30^{\circ}$, with respect to the image SI axis), in agreement with previous studies $(26,40)$. Similarly $(41,42)$, we measured the internal-external phase shift, as it can affect surrogatedriven motion compensation strategies. Specifically, in the study by Gierga et al (41), fluoroscopic imaging was used to detect internal and external motion in 4 liver patients in which phase shifts were not observed, whereas the study of 5 patients by Nishioka et al (42) relied on fluoroscopy combined with an external monitoring de-vice, measuring limited phase shifts (up to 0.09

$\pi=16.2^{\circ}$ ). In our work, 11 subjects showed comparable results, as their phase shifts were limited in all detected liver features (25th and 75th percentiles within $\pm 8^{\circ}$ ), whereas larger shifts were observed in the remaining subjects. Such a variability can be due to the different location of the oblique slices with respect to the liver, whereas the intrasubject variability can be attributed to the wide features spatial distribution.

The cine-MRI method allows accurate measurement of the motion amplitude even in the presence of breathing irregularities, which can be fully quantified and do not cause artifacts as in 4DCT (43). Finally, relevant inter- and intrasubject variations were observed also in motion amplitude (Fig. 2 C), thus confirming the potential advantages of subject-specific and spatially distributed measurements. Interfractional variability of these measurement could also be investigated by acquiring multiple sets of cineMRI images on different days.

\section{Surrogate-based versus MRI-guided tracking}

We quantified the potential gain of MRI guidance versus that with the use of external and internal correlation models to estimate the motion of specific liver features $\left(M R I_{\text {gain }}\right)$. We therefore derived an experimental curve to correlate the expected gain of MRI guidance as a function of the required localization accuracy (ie the tracking error threshold $\mathrm{TE}_{\mathrm{th}}$ ) (Fig. 3). Such a curve can therefore be used to estimate the potential improvement in the application of MRI guidance, once a specific error threshold has been established as a clinical requirement. On average, the po-tential gain is limited (below 10\%) if the required accuracy is on the order of $3 \mathrm{~mm}$, with a steep increase when the error threshold is reduced to 1 to $2 \mathrm{~mm}$ (Fig. 3). In addition to average values, a large variability was observed, thus confirming that the performance of external/internal cor-relation models is extremely dependent on specific motion features, as discussed in the following section. Even if in this work the $M R I_{\text {gain }}$ was computed for each subject considering multiple liver features, the same index could be easily measured for a single feature or for a limited liver 

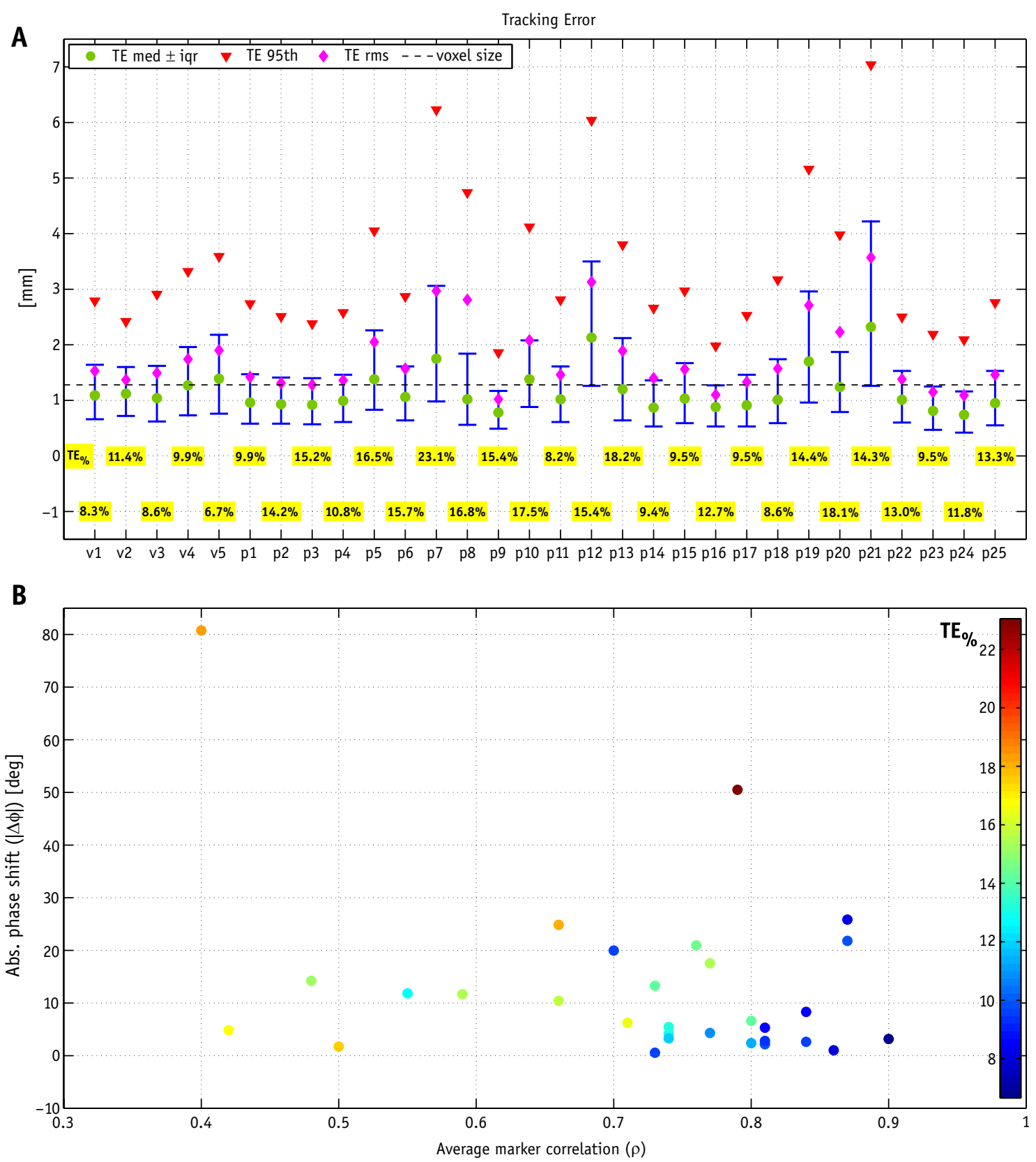

Fig. 4. (A) Tracking error expressed as $\mathbf{T} E_{\text {med }}^{\mathbf{s}} \pm \mathbf{T} E_{\text {iqr }}^{\mathbf{s}}$ (green circles and blue bars), $\mathbf{T} E_{95}^{\mathbf{s}}$ (red squares), $\mathbf{T} E_{\text {rms }}^{\mathbf{s}}$ (magenta diamonds), and $\mathbf{T} \mathbf{E}_{\%}^{\mathrm{s}}$ (yellow boxes). (B) Scatter plots representing $\mathbf{T} \mathbf{E}_{\%}^{\mathrm{s}}$ (color map from dark blue to dark red) as a function of absolute phase shift and average correlation between markers and features motion.

region (ie the tumor volume), according to specific clinical requirements.

It is worth considering that the relationship between $M R I_{\text {gain }}$ and error threshold was computed in the time frame of the reported cine-MRI acquisition (74.4-second duration), with no retraining of the external/internal correlation model. The performance of such models would benefit by the application of specific retraining protocols, as currently applied clinically in commercial systems implementing model-based motion tracking $(8,39)$. Also, the application of external/internal correlation models might be useful to complement MRI guidance in terms of out-of-plane motion estimation. Specifically, motion in the perpendicular direction with respect to the selected imaging plane could be estimated based on a dedicated model, thus providing full 3D information for motion tracking.

\section{Surrogate-based tracking performance}

Relying on the available cine-MRI dataset, the performance of external/internal correlation models was measured during a period of about 50s and in correspondence to each 

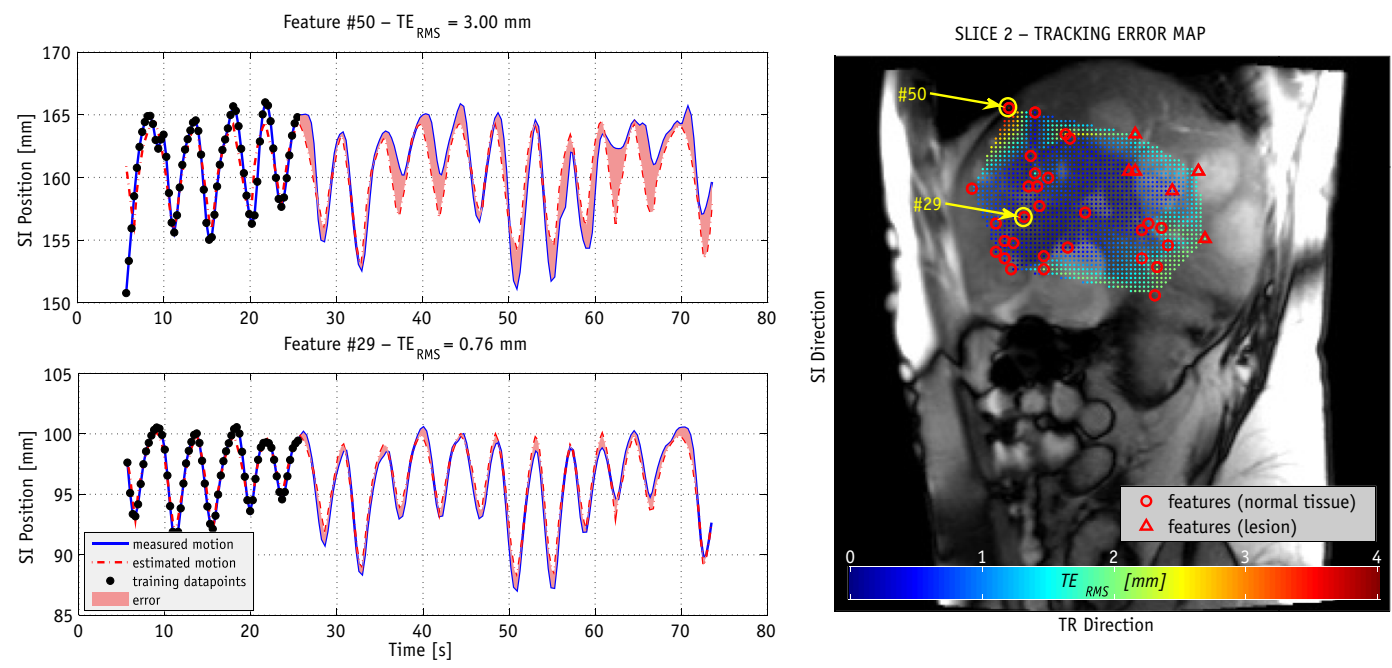

Fig. 5. (Right) Tracking error map (p14, slice 2). (Left) Measured (SIFT) and estimated (correlation model) motion traces are shown for feature nos. 50 and 29. Patient 14 was selected because large tracking error variability was observed within slice 2. SIFT $=$ scale invariant feature transform.

liver feature, as shown in the time-resolved TE map (Fig. E1, available at www.redjournal.org). Tracking errors were comparable ( $2 \mathrm{D}$ error 90th percentile $=2.72 \mathrm{~mm}$ ) to that reported by Depuydt et al (39) for the same correlation model applied to liver lesions (2D error 90th percentile $=3.14 \mathrm{~mm}$ ).

We found a statistically significant correlation of performance with different variables: the overall tracking accuracy $T E_{\%}^{s}$ correlated with phase shifts between external and internal motion, as already observed in (44). The average marker correlation with the internal motion $(\rho)$ had a stronger positive influence on tracking accuracy than the marker number $\left(R_{\rho}=-0.62, R_{m}=-0.47\right.$, respectively), suggesting that the accuracy of surrogate-driven motion mitigation strategies could be more effectively improved with patientspecific marker configurations, which could be optimized relying on information provided by cine-MRI patient specific studies. As a matter of fact, dedicated cine-MRI acquisition protocols can be envisioned, similarly to what was reported in the current study, to analyze the best approach for motion management in a case-specific fashion. As automated feature detection is available (such as SIFT-based methods), the detailed study of internal motion and external/internal correlation is possible and could be part of the treatment planning routine to select those cases where MRI guidance is essential to achieve the required accuracy.

\section{Conclusions}

The use of cine-MRI in combination with an automatic feature extraction method can be considered a valuable tool for motion-compensated liver radiation therapy as it could support standard 4DCT-based planning by providing spatially distributed and artifact-free motion measurements.
The proposed method could also provide a decision-making procedure to prospectively distinguish between patients that can be effectively treated with surrogate-based tracking methods and those that will require direct target tracking by means of in-room MRI guidance.

\section{References}

1. Seco J, Sharp GC, Wu Z, et al. Dosimetric impact of motion in free-breathing and gated lung radiotherapy: A 4D Monte Carlo study of intrafraction and interfraction effects. Med Phys 2008;35: 356-366.

2. Sawant A, Keall P, Pauly KB, et al. Investigating the feasibility of rapid MRI for image-guided motion management in lung cancer radiotherapy. BioMed Res Int 2014;2014:485067.

3. Hanley J, Debois MM, Mah D, et al. Deep inspiration breath-hold technique for lung tumors: The potential value of target immobilization and reduced lung density in dose escalation. Int J Radiat Oncol Biol Phys 1999;45:603-611.

4. Kubo HD, Hill BC. Respiration gated radiotherapy treatment: A technical study. Phys Med Biol 1996;41:83-91.

5. Keall PJ, Kini VR, Vedam SS, et al. Motion adaptive X-ray therapy: a feasibility study. Phys Med Biol 2001;46:1-10.

6. Keall PJ, Mageras GS, Balter JM, et al. The management of respiratory motion in radiation oncology report of AAPM Task Group 76. Med Phys 2006;33:3874-3900.

7. McClelland JR, Hawkes DJ, Schaeffer T, et al. Respiratory motion models: A review. Med Image Anal 2012;17:19-42.

8. Kilby W, Dooley JR, Kuduvalli G, et al. The CyberKnife robotic radiosurgery system in 2010. Technol Cancer Res Treat 2010;9:452.

9. Verellen D, Depuydt T, Gevaert T, et al. Gating and tracking, 4D in thoracic tumours. Cancer Radiother 2010;14:446-454.

10. Dawson LA, Sharpe MB. Image-guided radiotherapy: Rationale, benefits, and limitations. Lancet Oncol 2006;7:848-858.

11. Jaffray DA. Image-guided radiotherapy: From current concept to future perspectives. Nat Rev Clin Oncol 2012;9:688-699.

12. Keall PJ. 4-Dimensional computed tomography imaging and treatment planning. Semin Radiat Oncol 2004;14:81-90.

13. Kauczor HU, Plathow C. Imaging tumour motion for radiotherapy planning using MRI. Cancer Imaging 2006;6:140-144. 
14. Mutic S, Dempsey JF. The ViewRay system: Magnetic resonanceguided and controlled radiotherapy. Semin Radiat Oncol 2014;24: 196-199.

15. Fallone BG. The rotating biplanar linac-magnetic resonance imaging system. Semin Radiat Oncol 2014;24:200-202.

16. Keall PJ, Barton M, Crozier S. The Australian magnetic resonance imaging-linac program. Semin Radiat Oncol 2014;24:203-206.

17. Lagendijk JJW, Raaymakers BW, van Kulpen M. The magnetic reso-nance imaging-linac system. Semin Radiat Oncol 2014;24:207-209.

18. Tryggestad E, Flammang A, Hales R, et al. 4D tumor centroid tracking using orthogonal 2D dynamic MRI: Implications for radiotherapy planning. Med Phys 2013;40:091712.

19. Bierre T, Crijns S, af Rosenschöld PM, et al. Three-dimensional MRIlinac intra-fraction guidance using multiple orthogonal cine-MRI planes. Phys Med Biol 2013;58:4943-4950.

20. Kupelian P, Sonke JJ. Magnetic resonance-guided adaptive radiotherapy: A solution to the future. Semin Radiat Oncol 2014;24: 227-232.

21. Plathow C, Ley S, Fink C, et al. Analysis of intrathoracic tumor mobility during whole breathing cycle by dynamic MRI. Int J Radiat Oncol Biol Phys 2004;59:952-959.

22. Plathow C, Klopp M, Fink C, et al. Quantitative analysis of lung and tumour mobility: Comparison of two time-resolved MRI sequences. Br J Radiol 2005;78:836-840.

23. Roholfing T, Maurer CR, O'Dell WG, et al. Modeling liver motion and deformation during the respiratory cycle using intensity-based nonrigid registration of gated MR images. Med Phys 2004;31:427-432.

24. Koch N, Liu HH, Starkschall G, et al. Evaluation of internal lung motion for respiratory-gated radiotherapy using MRI: Part I-Correlating internal lung motion with skin fiducial motion. Int $J$ Radiat Oncol Biol Phys 2004;60:1459-1472.

25. Liu HH, Koch N, Starkschall G, et al. Evaluation of internal lung motion for respiratory-gated radiotherapy using MRI: Part II-Margin reduction of internal target volume. Int J Radiat Oncol Biol Phys 2004;60:1473-1483.

26. Kirilova A, Lockwood G, Choi P, et al. Three-dimensional motion of liver tumors using cine-magnetic resonance imaging. Int $J$ Radiat Oncol Biol Phys 2008;71:1189-1195.

27. Song R, Tipirneni A, Johnson P, et al. Evaluation of respiratory liver and kidney movements for MRI navigator gating. J Magn Reson Imaging 2011;33:143-148.

28. Dowling JA, Dang K, Chris DF, et al. Fast cine-magnetic resonance imaging point tracking for prostate cancer radiation therapy planning. J Phys Conf Ser 2014;489:012027.

29. Brix L, Ringgaard S, Sørensen TS, et al. Three-dimensional liver motion tracking using real-time two-dimensional MRI. Med Phys 2014;41:042302.
30. Cervino LI, Du J, Jiang SB. MRI-guided tumor tracking in lung cancer radiotherapy. Phys Med Biol 2011;56:3773-3785.

31. Shi X, Diwanji T, Mooney KE, et al. Evaluation of template matching for tumor motion management with cine-MR images in lung cancer patients. Med Phys 2014;41:052304.

32. Allaire S, Kim JJ, Breen SL, et al. Full orientation invariance and improved feature selectivity of 3D SIFTwith application to medical image analysis. IEEE Computer Society Conference on Computer Vision and Pattern Recognition Workshops. June 23-28. Anchorage, AK 2008, 1-8.

33. Paganelli C, Peroni M, Riboldi M, et al. Scale invariant feature transform in adaptive radiation therapy: A tool for deformable image registration assessment and re-planning indication,. Phys. Med. Biol. 2013;58:287-299.

34. Paganelli C, Peroni M, Pennati F, Baroni G, Summer P, Bellomi M, Riboldi M. Scale invariant feature transform as feature tracking method in 4D imaging: A feasibility study,. 34th Annual International Conference of the IEEE (EMBS) 2012;1:6543-6546.

35. Paganelli C, Peroni M, Baroni G, Riboldi M. Quantification of organ motion based on an adaptive image-based scale invariant feature method. Med Phys. 2013;40:111701.

36. Ioannoua D, Hudab W, Lainec AF, et al. Circle recognition through a 2D Hough transform and radius histogramming. Image Vis Comput 1999;17:15-26.

37. Nuclear Associates 76-907 and 76-908 AAPM MRI Phantoms, Fluke Corporation Radiation Management Services 2005.

38. P. Berens, CircStat: A Matlab Toolbox for Circular Statistics, Journal of Statistical Software, Volume 31, Issue 10, pp. 1-21, 2009

39. Depuydt T, Poels K, Verellen D, et al. Initial assessment of tumor tracking with a gimbaled linac system in clinical circumstances: A patient simulation study. Radiother Oncol 2013;106:236-240.

40. Park JC, Park SH, Kim JH, et al. Liver motion during cone beam computed tomography guided stereotactic body radiation therapy. Med Phys 2012;39:6431-6442.

41. Gierga DP, Brewer J, Sharp GC, et al. The correlation between internal and external markers for abdominal tumors: Implications for respiratory gating. Int J Radiat Oncol Biol Phys 2005;61:1551-1558.

42. Nishioka T, Nishioka S, Kawahara M, et al. Synchronous monitoring of external/internal respiratory motion validity of respiration-gated radiotherapy for liver tumors. Jpn J Radiol 2009;27:285-289.

43. Yamamoto T, Langner $\mathrm{U}, \mathrm{Loo} \mathrm{BW}$. Retrospective analysis of artifacts in four-dimensional CT images of 50 abdominal and thoracic radiotherapy patients. Int $J$ Radiat Oncol Biol Phys 2008;72:1250-1258.

44. Seregni M, Cerveri P, Riboldi M, Pella A, Baroni G. Robustness of external/internal correlation models for real-time tumor tracking to breathing motion variations. Phys Med Biol. 2012;57: 7053-7074. 